Article

\title{
Viscoelastic Effects on the Entropy Production in Oscillatory Flow between Parallel Plates with Convective Cooling
}

\author{
Federico Vázquez ${ }^{1}$, Miguel Ángel Olivares-Robles ${ }^{2, \star}$ and Sergio Cuevas ${ }^{3}$ \\ ${ }^{1}$ Departamento de Fisica, Facultad de Ciencias, UAEM, Cuernavaca, Morelos, 62209, Mexico \\ ${ }^{2}$ Seccion de Estudios de Posgrado e Investigacion, ESIME-Culhuacan, Instituto Politecnico \\ Nacional, 04430, Mexico \\ ${ }^{3}$ Departamento de Termociencias, Centro de Investigacion en Energia, Universidad Nacional \\ Autonoma de Mexico, A. .P. 34, Temixco, Morelos 62580, Mexico
}

E-mails: vazquez@buzon.uaem.mx; olivares67@mailaps.org; scg@cie.unam.mx

* Author to whom correspondence should be addressed.

Received: 13 November 2008/Accepted: 22 December 2008/ Published: 28 December 2008

\begin{abstract}
The heat transfer problem of a zero-mean oscillatory flow of a Maxwell fluid between infinite parallel plates with boundary conditions of the third kind is considered. With these conditions, the amount of heat entering or leaving the system depends on the external temperature as well as on the convective heat transfer coefficient. The local and global timeaveraged entropy production are computed, and the consequences of convective cooling of the plates are also assessed. It is found that the global entropy production is a minimum for certain suitable combination of the physical parameters. For a discrete set of values of the oscillatory Reynolds number, the extracted heat from one of the plates shows maxima.
\end{abstract}

Keywords: Entropy generation; Optimization; Oscillatory flows; boundary conditions of the third type.

\section{Introduction}

In energy conversion processes, the performance of real thermal devices is always affected by irreversible losses that lead to an increase of entropy and reduce the thermal efficiency. Therefore, a great 
deal of effort has been carried out in order to understand the underlying physical processes with the aim at reducing such irreversibilities according to the physical constraints imposed on the system. On the other hand, the design of many traditional engineering devices (such as heat exchangers and cooling modules) and the performance of processes involving heat removal from components such as electronic chips and other similar high energy devices rely on heat transfer enhancement. In the previous situations, the analysis of the entropy generation $[1,2]$ reveals as a tool for characterizing the associated irreversible processes and, hopefully, optimizing the performance of a given device. In fluid flow systems, the entropy generation usually involves irreversibilities originated by heat transfer and viscous friction. It has been recognized that oscillatory flows may play an important role on the improvement of transport processes in different situations. For instance, oscillatory flows may enhance the transport of mass compared to the molecular diffusive transport present in a motionless fluid [3]. In a similar way, the effective thermal diffusivity of a Newtonian fluid in a duct subjected to a zero-mean oscillatory flow may reach a maximum for a specific oscillation frequency and lead to heat transfer enhancement $[4,5]$. Moreover, in the case of a viscoelastic fluid flowing in a tube, the dynamic permeability may be enhanced at given resonant oscillation frequencies [6, 7, 9-11]. Recently, attention has been directed to oscillatory flows at high frequencies under conditions where inertial effects are negligible with the aim at using them in microfluidics applications [12]. Some other recent studies have also addressed the problem of heat transfer with viscoelastic fluids [13-15]. Thus, one may reasonably wonder whether one can profit from a sensible combination of oscillatory flows, thermal boundary conditions and viscoelastic properties of the working fluid. This paper is devoted to providing an analysis of the heat transfer problem of a zero-mean oscillatory flow of a Maxwell fluid between infinite parallel plates with thermal boundary conditions based on Newton's law of cooling where the amount of heat entering or leaving the system depends on the external temperature as well as on the convective heat transfer coefficient. Such an analysis will hopefully shed some light on the heat transfer enhancement process. The emphasis is placed on the computation of the entropy generation for the system and its subsequent analysis [16].

\section{Transport Problem}

\subsection{Basic Assumptions}

We consider the flow of a viscoelastic fluid, modeled as a Maxwell fluid, between two infinite parallel plates separated by a distance $2 a$, so that border effects are disregarded. We assume that a zero-mean time-periodic pressure gradient is established in the system producing an oscillatory flow in the axial $x$-direction that satisfies nonslip conditions at the plates. With the former approximations, the flow becomes fully developed with all quantities depending on the transversal coordinate $y$ and the time $t$, except for the pressure, which varies with $x$ and $t$. Provided the flow remains within the linear regime assumptions, the stability of the flow is assured. We also assume that the fluid is incompressible and monocomponent, so that mass diffusion phenomena are disregarded. In addition, all physical properties of the fluid are assumed to be constant. Heat is generated in the fluid due to viscous stresses and it is assumed that the system exchanges heat with the exterior through Newton's law of cooling so that the heat transfer equation must satisfy thermal boundary conditions of the third kind. 


\subsection{Fundamental Equations}

The continuity and momentum equations for this system are

$$
\begin{gathered}
\nabla \cdot \mathbf{u}=0 \\
\rho\left(\frac{\partial \mathbf{u}}{\partial t}+(\mathbf{u} \cdot \nabla) \mathbf{u}\right)=-\nabla p+\nabla \cdot \boldsymbol{\tau}
\end{gathered}
$$

where $\mathbf{u}$ and $p$, are the velocity and pressure fields while $\rho$ and $\boldsymbol{\tau}$ are the mass density and viscous stress tensor of the fluid. The right-hand side of Eq. (2) accounts for normal and tangential stresses, respectively. In turn, the heat transfer equation reads

$$
\rho c_{p}\left(\frac{\partial T}{\partial t}+(\mathbf{u} \cdot \nabla) T\right)=k \nabla^{2} T-\boldsymbol{\tau}: \nabla \mathbf{u},
$$

where $T$ is the temperature, and $c_{p}$ and $k$ are the specific heat at constant pressure and the thermal conductivity of the fluid, respectively. For the viscous stress tensor $\tau$ we consider the linear form of the Maxwell model, namely,

$$
t_{m} \frac{\partial \boldsymbol{\tau}}{\partial t}=-\eta \nabla \mathbf{u}-\boldsymbol{\tau}
$$

where $\eta$ is the shear viscosity and $t_{m}$ the relaxation time for the Maxwell fluid. Note that if $t_{m}=0$ we recover the expression for the viscous stress tensor for the Newtonian fluid given by

$$
\boldsymbol{\tau}=-\eta \nabla \mathbf{u}
$$

\subsection{Velocity field}

In order to get the governing equation of motion, we first take the time derivative of equation (2) without the nonlinear convective term. Then, we substitute the term $\partial \tau / \partial t$ from equation (4) and use again equation (2) to eliminate the term $\nabla \cdot \tau$. Assuming a unidirectional flow that depends only on the transversal coordinate and time, the final equation reads

$$
t_{m} \rho \frac{\partial^{2} u}{\partial t^{2}}+\rho \frac{\partial u}{\partial t}=-\left(1+t_{m} \frac{\partial}{\partial t}\right) \frac{\partial p}{\partial x}+\eta \frac{\partial^{2} u}{\partial y^{2}} .
$$

The oscillatory pressure gradient that produces the motion can be expressed as the real part of $(\partial p / \partial x)=$ $G e^{i \omega t}$, where $G$ is a constant. Therefore, we assume that the velocity is also a harmonic function of $t$, that is, $\mathbf{u}=\left[u=\frac{1}{2}\left(u_{o}(y) e^{i \omega t}+\overline{u_{o}(y)} e^{-i \omega t}\right), 0,0\right]$, where the bar denotes complex conjugation. Under these circumstances the equation to be solved reads

$$
\frac{\partial^{2} u_{0}}{\partial y^{* 2}}=\left(1+i \omega^{*}\right)\left(i R_{\omega} u_{0}+K\right)
$$

where $y^{*}=y / a, K=G a^{2} / \eta, R_{\omega}=\omega a^{2} \rho / \eta$ being the oscillation Reynolds number (which compares the characteristic length with the viscous penetration depth) and $\omega^{*}=\omega t_{m}$. The corresponding non-slip boundary conditions for eq. (7) read $u_{0}( \pm 1)=0$. The average velocity $U_{0}$ is in turn given by

$$
U_{o}=\frac{1}{2} \int_{-1}^{+1} u_{o} d y^{*} .
$$


The explicit (analytic) expression of $u_{0}\left(y^{*}\right)$ in terms of $R_{\omega}, K$ and $\omega^{*}$ may be readily derived from Eq. (7) and its boundary conditions (as we have done using Mathematica) but will not be written down here.

\subsection{Temperature field}

Once the velocity field is obtained, one may proceed to solve the energy balance equation. Here this equation must be solved using boundary conditions of the third kind that indicate that the normal temperature gradient at any point in the boundary is assumed to be proportional to the difference between the temperature at the surface and the external ambient temperature. Hence, the amount of heat entering or leaving the system depends on the external temperature as well as on the convective heat transfer coefficient. It is also useful to consider the dimensionless variables $u^{*}=u / U_{o}$ and $t^{*}=\omega t$. Then, the energy balance (3) for the Maxwell fluid may be written, in dimensionless form, as

$$
\operatorname{Pr} R_{\omega} \frac{\partial \Theta}{\partial t^{*}}=\frac{\partial^{2} \Theta}{\partial y^{* 2}}+\frac{1}{1+\omega^{* 2}}\left(\frac{\partial u^{*}}{\partial y^{*}}\right)^{2}
$$

where $\operatorname{Pr}=\eta c_{p} / k$, is the Prandtl number and the dimensionless temperature is given by $\Theta=k T / \eta U_{o}^{2}$. Note that the viscous dissipation contribution involves squared terms of harmonic functions of time. Consequently, the heat source term contains time harmonic terms with twice the frequency of oscillation as well as a steady contribution. Therefore, one may assume that the dimensionless temperature has the form

$$
\Theta\left(y^{*}, t^{*}\right)=\Theta_{u}\left(y^{*}\right) e^{2 i t^{*}}+\overline{\Theta_{u}\left(y^{*}\right)} e^{-2 i t^{*}}+\Theta_{s}\left(y^{*}\right),
$$

where the sub-indexes $u$ and $s$ refer to the unsteady and steady contributions, respectively. Introducing Eq.(10) into Eq. (9), the equations satisfied by $\Theta_{u}, \overline{\Theta_{u}}$ and $\Theta_{s}$ are found to be

$$
\begin{aligned}
& \frac{d^{2} \Theta_{u}}{d y^{* 2}}-2 i \operatorname{Pr} R_{\omega} \Theta_{u}=-\frac{1}{4\left(1+\omega^{* 2}\right)}\left(\frac{\partial u_{0}^{*}}{\partial y^{*}}\right)^{2}, \\
& \frac{d^{2} \overline{\Theta_{u}}}{d y^{* 2}}+2 i \operatorname{Pr} R_{\omega} \overline{\Theta_{u}}=-\frac{1}{4\left(1+\omega^{* 2}\right)}\left(\frac{\partial \overline{u_{0}^{*}}}{\partial y^{*}}\right)^{2},
\end{aligned}
$$

and

$$
\frac{d^{2} \Theta_{s}}{d y^{* 2}}=-\frac{1}{2\left(1+\omega^{* 2}\right)}\left(\frac{\partial u_{0}^{*}}{\partial y^{*}}\right)\left(\frac{\partial \overline{u_{0}^{*}}}{\partial y^{*}}\right) .
$$

The solution to Eqs. (11)-(13) must satisfy the boundary conditions of the third kind, namely

$$
\begin{gathered}
\frac{d \Theta_{s}}{d y^{*}}+B i_{1}\left(\Theta_{s}-\Theta_{A}\right)=0, \quad \text { at } \quad y^{*}=1, \\
\frac{d \Theta_{s}}{d y^{*}}-B i_{2}\left(\Theta_{s}-\Theta_{A}\right)=0, \quad \text { at } \quad y^{*}=-1, \\
\frac{d \Theta_{u}}{d y^{*}}+B i_{1} \Theta_{u}=0, \quad \text { at } \quad y^{*}=1, \\
\frac{d \Theta_{u}}{d y^{*}}-B i_{1} \Theta_{u}=0, \quad \text { at } \quad y^{*}=-1,
\end{gathered}
$$


Figure 1. Dimensionless velocity vs. dimensionless position $y^{*}$ at $t^{*}=0.5$. Solid line: Maxwell fluid, $R_{\omega}=1, \omega^{*}=1.8 \times 10^{5}$, dot-dashed line: Newtonian fluid, $R_{\omega}=1$.

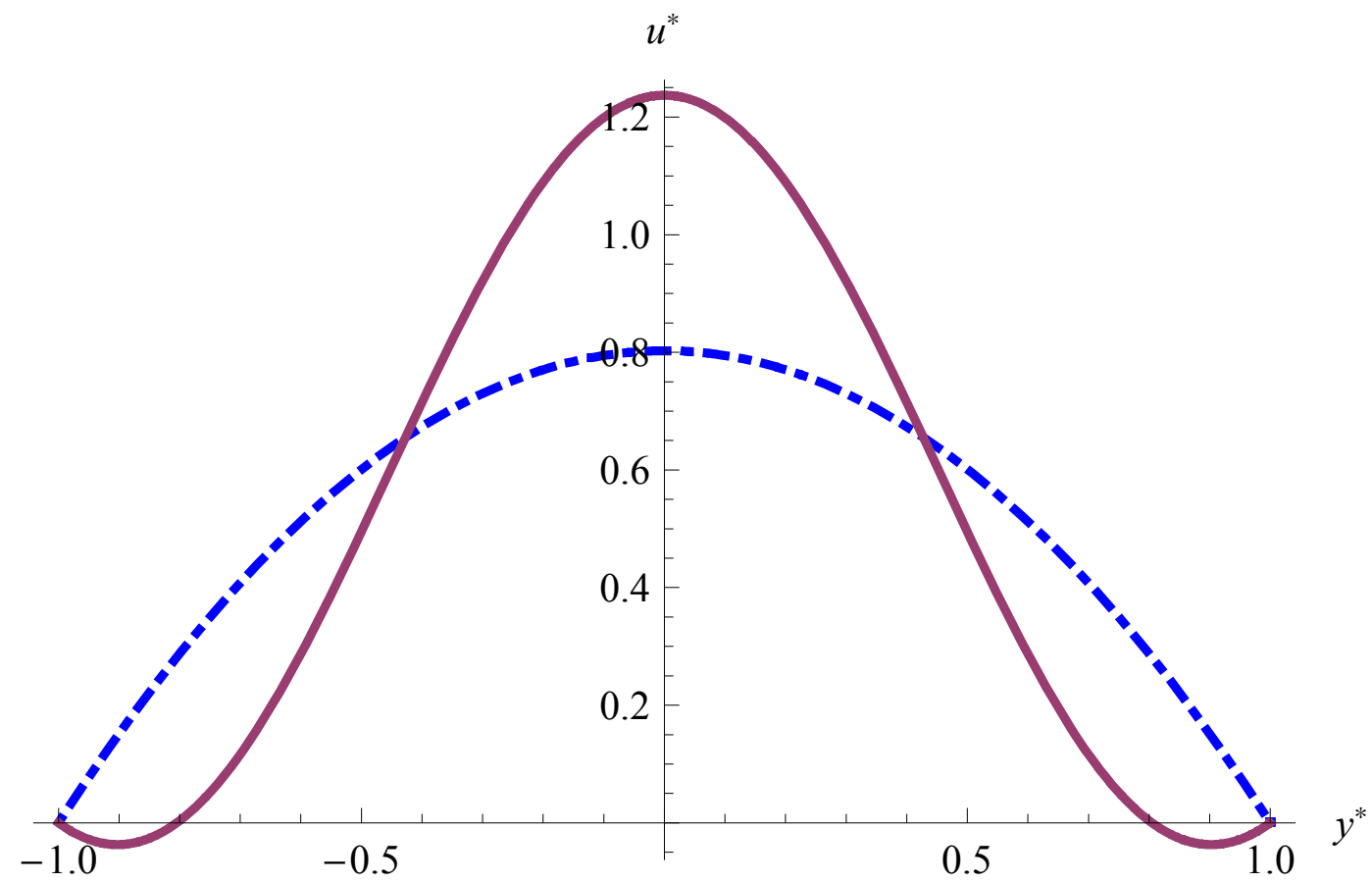

where $\Theta_{A}$ is the reference ambient dimensionless temperature and the Biot numbers $B i_{1}=h_{1} a / k$ and $B i_{2}=h_{2} a / k$ are the dimensionless expressions of the convective heat transfer coefficients of the upper and lower surfaces, $h_{1}$ and $h_{2}$, respectively, which can be different. As stated before, with these conditions the amount of heat entering or leaving the system depends on the external ambient temperature $\Theta_{A}$, as well as on the dimensionless convective heat transfer coefficient of each surface. When the Biot number tends to zero, an adiabatic boundary is approached. In turn, for very high values of Biot number, a constant temperature boundary is recovered. Apart from the spatial or the spatial and temporal dependence, such solutions (derived again with the aid of Mathematica), are functions of $P_{r}, R_{\omega}, B i_{1}$, $B i_{2}$ and $\omega^{*}$ whose explicit form is not particularly illuminating and thus will also be omitted. They are, however, available upon request. For the sake of illustration, Figures 1 and 2 show the cross variation of the velocity and temperature fields, respectively, for both the Maxwell and the Newtonian fluids in each case.

The Newtonian description has been obtained by making $t_{m}=0$ in Eqs. (7), (11)-(13). Note, in Figure 1, the middle zone between the plates where the Maxwell fluid flows with an inverted velocity with respect to that near the plates. A particular choice of the values of the parameters defining the system was made in order to perform these and the further simulations. The values are the following: $k=0.58 \mathrm{~W} / \mathrm{mK}, \rho=1050 \mathrm{Kg} / \mathrm{m}^{3}, c_{p}=4.2 \times 10^{3} \mathrm{~J} / \mathrm{KgK}$ and $\eta=60 \mathrm{Pas}, t_{m}=1.9 \mathrm{~s}$. The viscoelastic properties of the fluid correspond to an aqueous solution of cetylpyridinium chloride (CPyCl) $100 \mathrm{mM}$ and sodium salisylate (NaSal) $60 \mathrm{mM}$ [17].

The oscillation frequency $\omega$ is ranging from $6 \times 10^{2} \mathrm{~Hz}\left(R_{\omega}=10^{3}\right)$ to $9 \times 10^{6} \mathrm{~Hz}\left(R_{\omega}=15\right)$, for a milimetric device (a separation between plates of $10^{-3} \mathrm{~m}$ ). We have also taken $\operatorname{Pr}=100, \Theta_{A}=5$, and the following intervals for the remaining parameters: $0<B i_{1}<10,3<B i_{2}<60$. 
Figure 2. Dimensionless temperature vs. dimensionless position $y^{*}$ at $t^{*}=0.5 . \operatorname{Pr}=100$, $B i_{1}=1, B i_{2}=50, \Theta_{A}=10$. Solid line: Maxwell fluid $R_{\omega}=1, \omega^{*}=1.8 \times 10^{5}$, dot-dashed line: Newtonian fluid, $R_{\omega}=1$.

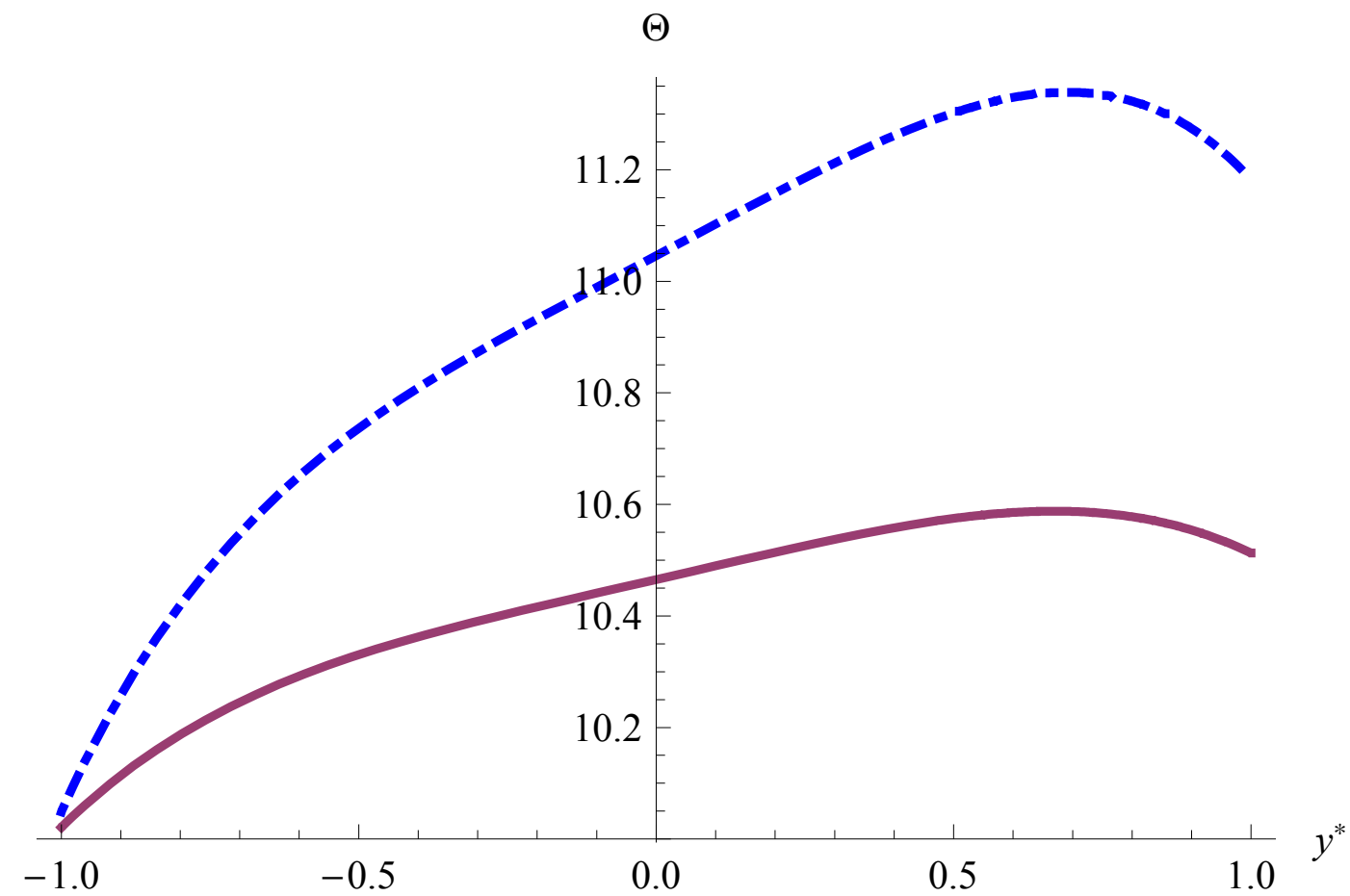

\section{Results}

\subsection{Entropy Generation}

The velocity and temperature fields already obtained will be used for the determination of the entropy generation rate for this problem. The heat transfer contribution to the dimensionless local entropy generation, $\dot{S}_{q}^{*}$, is not modified by the viscoelastic properties of the fluid and presents the usual form also valid for Newtonian fluids $[2,16]$, namely,

$$
\dot{S}_{q}^{*}=\frac{1}{\Theta^{2}}\left(\frac{\partial \Theta}{\partial y^{*}}\right)^{2} .
$$

On the other hand, the viscous stress contribution to the (dimensional) local entropy generation rate, $\dot{S}_{v}$, in general, is given by

$$
\dot{S}_{v}=-\boldsymbol{\tau}: \nabla \mathbf{u} / T
$$

For the Maxwellian case, the expression for $\dot{S}_{v}$ has to be derived by considering the constitutive equation for the stress tensor of the Maxwell fluid given by Eq. (4) [11]. Expressed in integral form, we have [18],

$$
\boldsymbol{\tau}(t)=-\int_{-\infty}^{t}\left[\frac{\eta}{t_{m}} e^{-\frac{t-t^{\prime}}{t_{m}}}\right] \nabla \mathbf{u}\left(t^{\prime}\right) d t^{\prime}
$$


Figure 3. Dimensionless global entropy generation rate $\dot{S}_{a v}^{*}$ vs $B i_{1}$. Maxwell fluid: $\operatorname{Pr}=$ 100, $R_{\omega}=10, \Theta_{A}=5, \omega^{*}=1.08 \times 10^{6}$. Solid line: $B i_{2}=60$ dot-dashed line: $B i_{2}=5$, dotted line: $B i_{2}=3$.

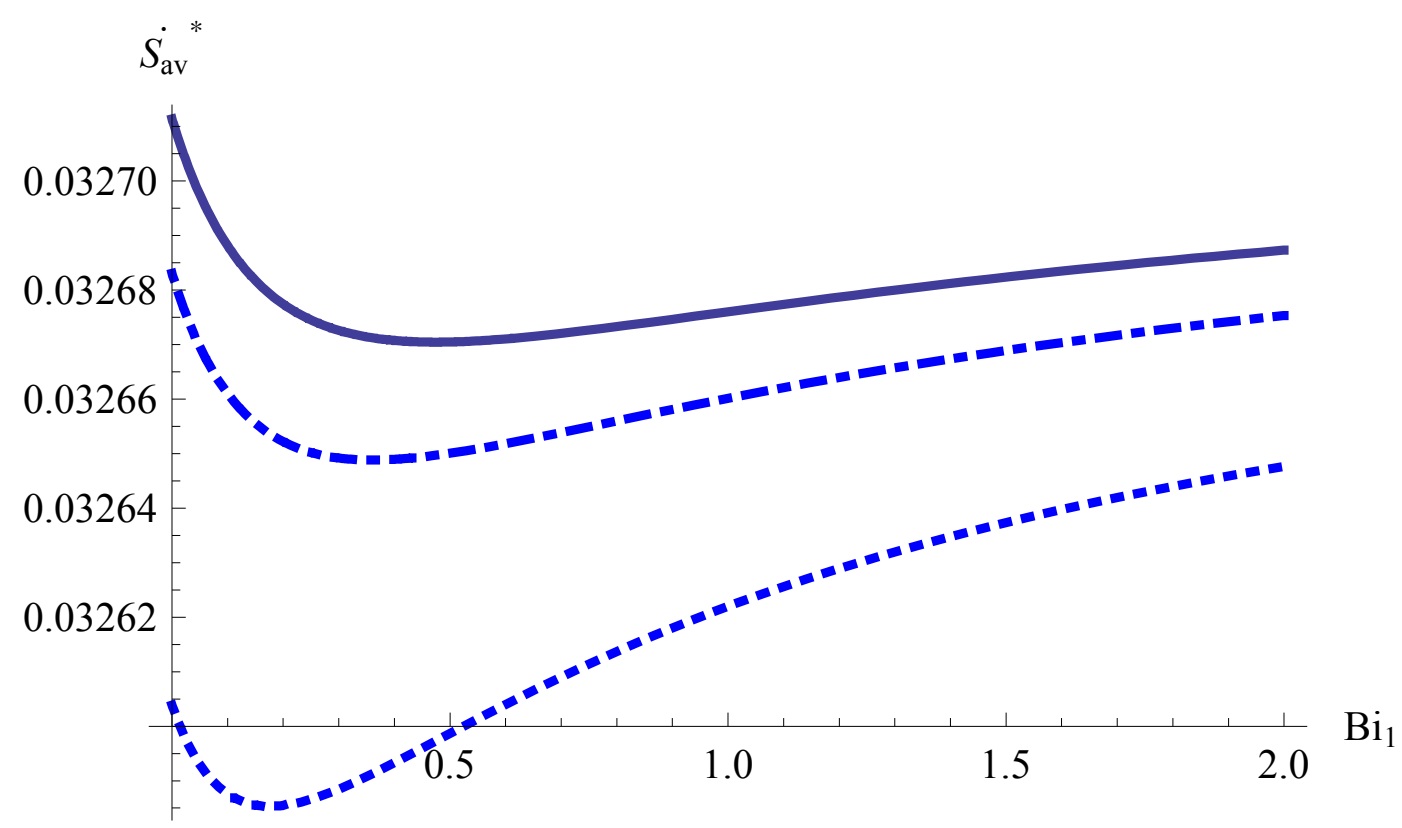

Then, we substitute Eq. (20) into Eq. (19) in order to obtain the general expression for viscous stress contribution of the entropy generation rate for a Maxwellian fluid, namely,

$$
\dot{S}_{v}=\left[-\int_{-\infty}^{t}\left[\frac{\eta}{t_{m}} e^{-\frac{t-t^{\prime}}{t_{m}}}\right] \nabla \mathbf{u}\left(t^{\prime}\right) d t^{\prime}\right]:[\nabla \mathbf{u}(t)] / T .
$$

Explicitly, once the velocity profile is substituted into Eq. (21) and the integral of $\dot{S}_{v}$ over one period of oscillation is calculated, we find the local (time-averaged) entropy generation rate for the Maxwellian fluid that, in dimensionless terms, reads

$$
<\dot{S}_{v}^{*}>\left(y^{*}\right)=\frac{1}{\Theta\left(1+\omega^{* 2}\right)}\left(\frac{\partial u_{0}^{*}}{\partial y^{*}}\right)^{2},
$$

where the symbol $<>$ denotes time integration over one period of oscillation. Therefore, the dimensionless time-averaged local entropy generation rate, $\left\langle\dot{S}^{*}\right\rangle$, that takes into account irreversibilities due to heat conduction and viscous losses is given by

$$
<\dot{S}^{*}>=<\dot{S}_{q}^{*}>+<\dot{S}_{v}^{*}>
$$

where $<\dot{S}^{*}>$ is normalized by $k / a^{2}$. Previously to the time integration, $\dot{S}^{*}$ is a function of space and time and, in fact, contains both time harmonic and steady parts. Once the time-averaging is performed, time harmonic terms disappear and only the steady, space-dependent terms remain. The global entropy generation rate per unit length in the axial direction, denoted as $\dot{S}_{a v}^{*}$, is obtained by integrating $<\dot{S}^{*}>$ from $y^{*}=-1$ to $y^{*}=1$. While one could attempt to derive the resulting (complicated) function of $\operatorname{Pr}$, $R_{\omega}, B i_{1}, B i_{2}$, and $\omega^{*}$, it seems much more practical to compute it numerically. 
Figure 4. Log plot of dimensionless global entropy production $\dot{S}_{a v}^{*}$ vs $R_{\omega} . B i_{1}=0.5$, $B i_{2}=60, \Theta_{A}=5$. Solid line: Maxwell fluid $(\operatorname{Pr}=100)$, dot-dashed line: Newtonian fluid $(\operatorname{Pr}=1)$.

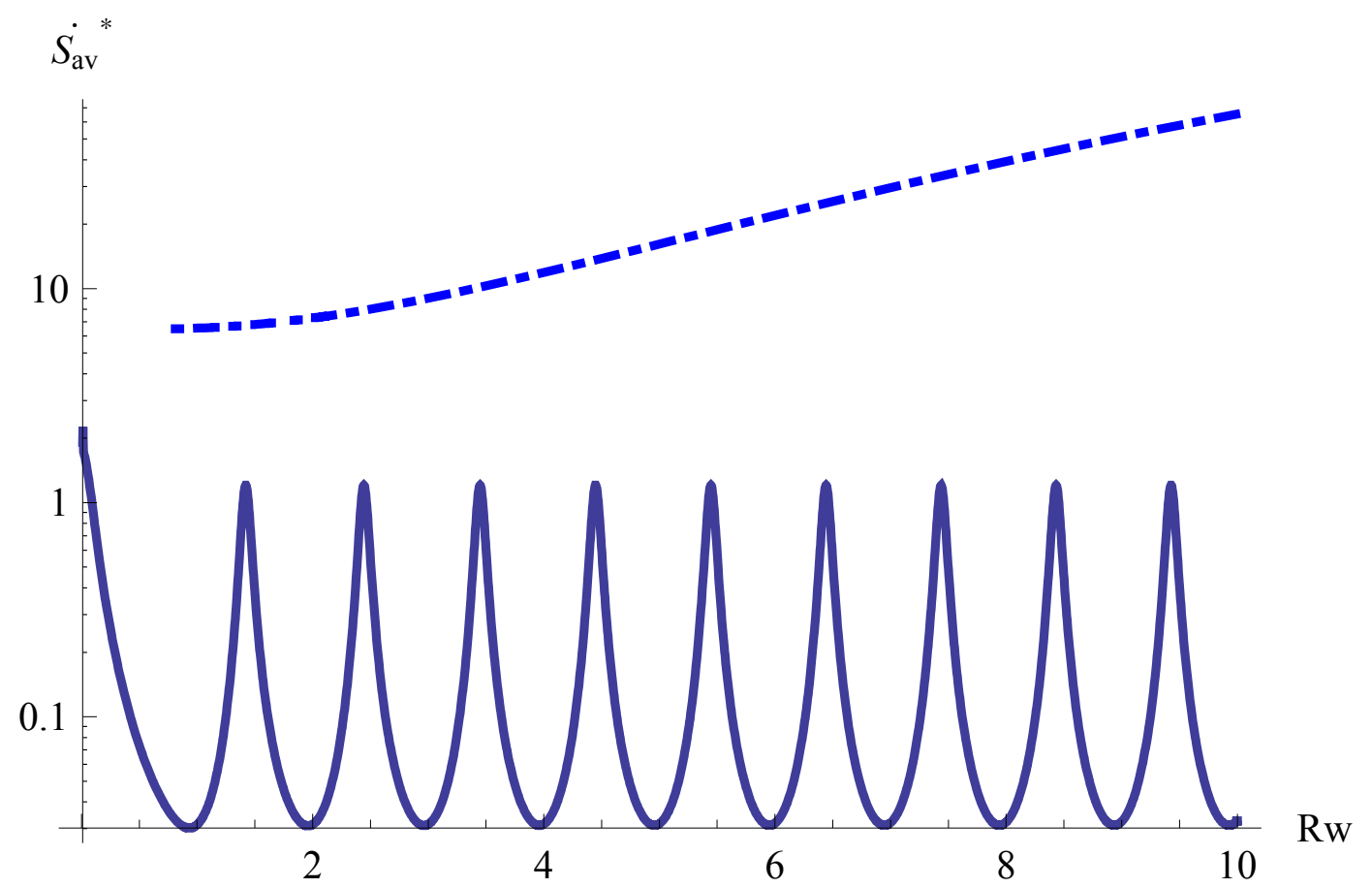

In Figure 3 we show the normalized global entropy generation rate, for the Maxwell fluid only, as a function of $B i_{1}$, for fixed $\operatorname{Pr}, R_{\omega}$ and $\omega^{*}$ and three different values of $B i_{2}$.

As clearly seen in Figure 3, the global entropy production displays well defined minima. A previous research has reported an analogous result for other heat transfer problem with a Newtonian fluid [19, 20]. These minima are due in part to the elastic properties of the Maxwell fluid manifested through the presence of relaxation terms in Eqs. $(7,11)-(13)$. Note that minima are only found for restricted values of the Biot numbers.

In the Log plot of Figure 4, it is displayed the behavior of the global entropy generation with respect to the oscillation Reynolds number for both the Newtonian and the Maxwell fluids. Unlike the Newtonian fluid, the dependence of $\dot{S}_{a v}^{*}$ on $R_{\omega}$ appears rather complicated in the case of the Maxwell fluid and it has a remarkable feature; namely, the global entropy production behaves periodically with $R_{\omega}$, so that for given frequencies the entropy generation reaches very small values. These low entropy production zones in $R_{\omega}$ should be considered in the design of thermal devices for the sake of optimal working conditions. Mention must be made that both curves in Figure 4 were obtained with the same values of the parameters, excepting the Prandtl number. The curves should be compared only in a qualitative way.

\subsection{Heat Transfer}

We now show the dependence of the normal heat transported through the parallel plates on both the Biot and the oscillatory Reynolds numbers. Firstly, we calculate the heat per unit area in a full oscillation period and the result for the lower plate can be seen in Figures 5 and 6, when plotted against $B i_{1}$ and 
Figure 5. Dimensionless heat transported per unit area in lower plate vs. $B i_{1}$. Solid line: Maxwell fluid, dot-dashed line: Newtonian fluid. $R_{\omega}=1.4, B i_{2}=60, \Theta_{A}=5, \omega^{*}=$ $1.51 \times 10^{6}$.

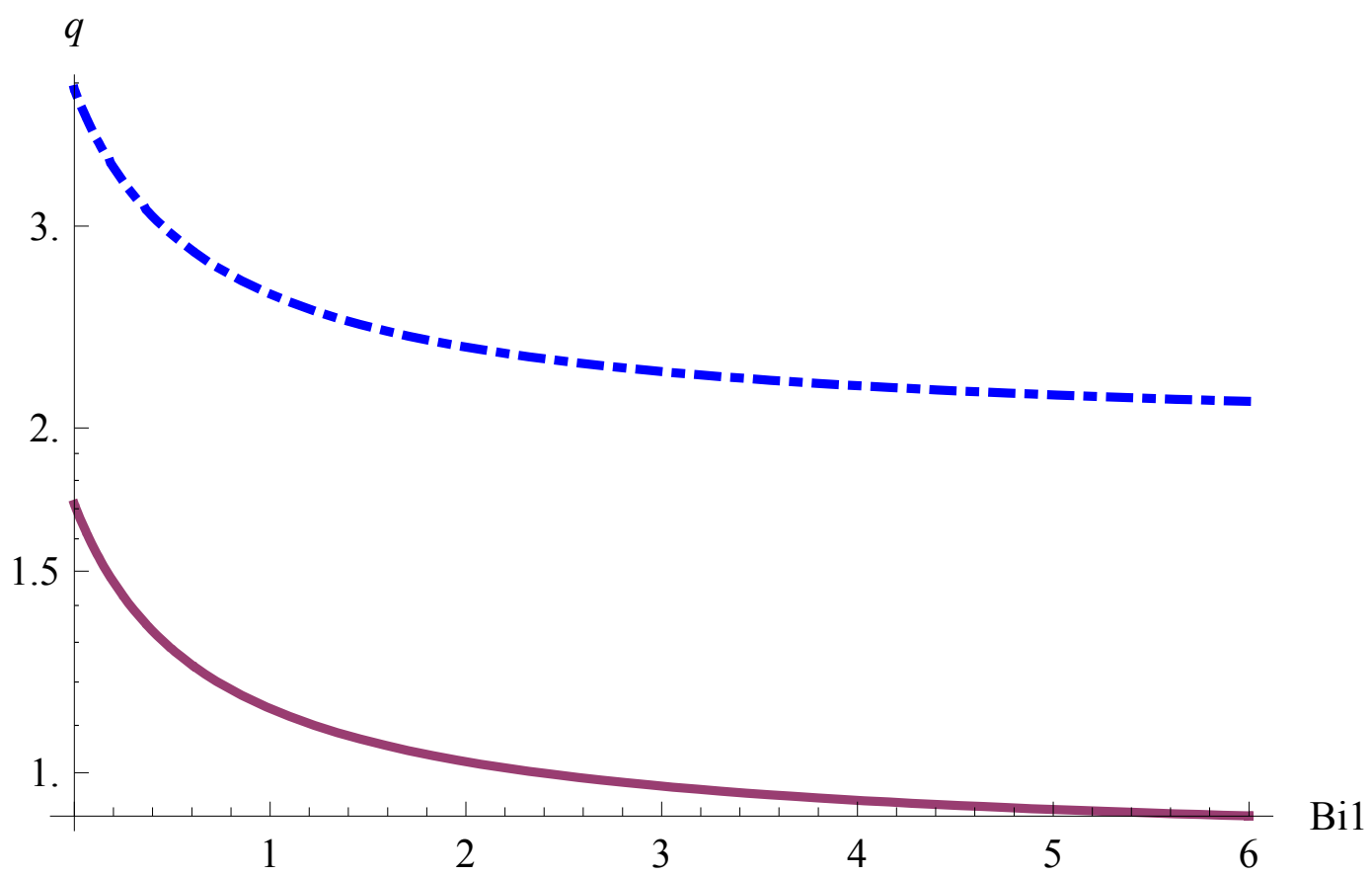

$B i_{2}$, respectively. In each one of those figures, we display the heat for both Maxwell and Newtonian fluids. We note that the graphs for both fluids can be compared in a quantitative way since they were obtained with exactly the same model parameters. Observe that the heat does not vanish as $B i_{1}$ goes to zero (Figure 5), while it does vanishes at $B i_{2}=0$ for the two fluids, as expected, since according with the boundary conditions, when $B i_{2}$ vanishes the heat flow is zero in the lower plate. We note the fact that the magnitude of the transported heat through the lower plate increases when $B i_{1}$ decreases (Figure 5 ) and when $B i_{2}$ increases (Figure 6). Though not displayed, the normal heat per unit area transferred through the upper plate shows a similar behavior.

Finally, we show in Figure 7 the transported heat through the lower plate as a function of $R_{\omega}$. Note firstly, that maxima of the heat exist where $\dot{S}_{a v}^{*}$ has also maxima, and secondly, that in all cases of Figures $5,6,7$, the transported heat flow is positive. It may be shown that the heat flow through the upper plate is also positive. Then, the device is extracting heat from the lower plate and giving a part of the absorbed or generated heat to the upper plate.

\section{Discussion}

The convective cooling of the parallel plates brings out a diminishing of the entropy production as the Biot number $B i_{1}$ increases. It reaches a minimum at certain $B i_{1}$ which depends on the value of $B i_{2}$, as for example, in the case when $B i_{2}=3$, the minimum appears at $B i_{1}=0.24$ (see Figure 3). The conditions of minimum entropy generation for the Maxwell fluid can then be sketched from Figures 3 and 4. In Figure 3, we see that minima exist if $0<B i_{1}<0.5$, when $B i_{2}$ is varying from 3 to 60 . On the 
Figure 6. Dimensionless heat transported per unit area in lower plate vs. $B i_{2}$ Solid line: Maxwell fluid, dot-dashed line: Newtonian fluid. $R_{\omega}=1.4, B i_{2}=60, \Theta_{A}=5, \omega^{*}=$ $1.51 \times 10^{6}$.

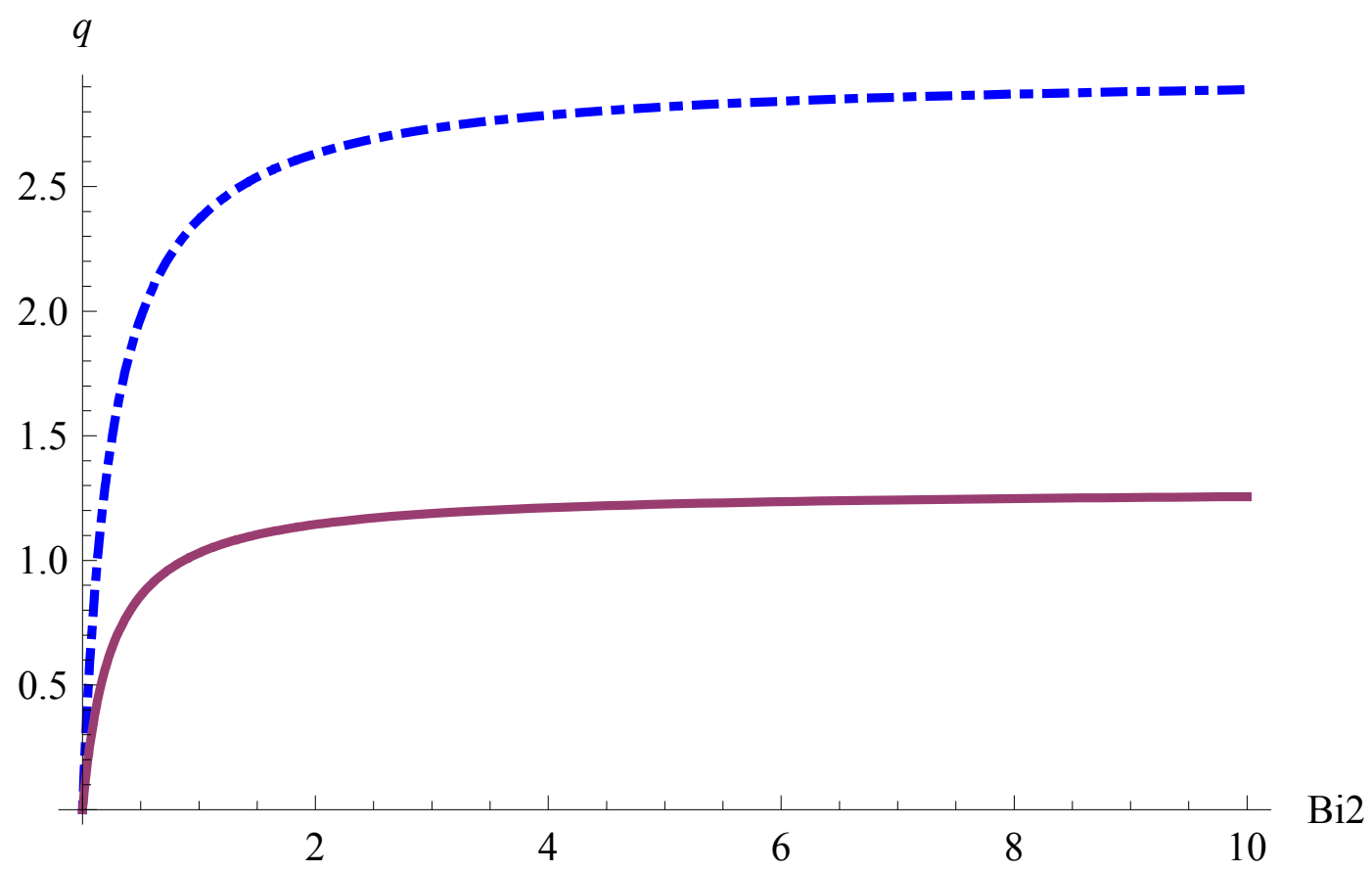

other hand, we periodically find minima of $\dot{S}_{a v}^{*}$ in the full range of $R_{\omega}$ considered in this study (Figure 4). The minima appear approximately at $R_{\omega}=1,2,3, .$. , and so on.

We remark the striking differences introduced by the peculiar properties of the Maxwell fluid in the entropy production analysis. As mentioned above, when $t_{m}=0$, the problem reduces to the one of the Newtonian fluid which is therefore a particular case of the present development. Our results suggest that the sensitivity of $\dot{S}_{a v}^{*}$ to a variation of $t_{m}$ would be very high. Far from the statement that the viscoelastic properties of the Maxwell fluid can make disappear the existence of minimal entropy production operating conditions, we see that, in fact, they make wider the spectrum of combinations of the parameters to obtain such conditions. From the results obtained in this work we have that given values of Biot numbers obeying $0.2<B i_{1}<0.5$ and $B i_{2}>1$, a suitable selection of $R_{\omega}$, practically in its whole range of variation, can bring out the minimal entropy production condition, which is not so in the case of the Newtonian fluid. In this case, the entropy production increases indefinitely as $R_{\omega}$ increases.

With respect to the heat transferred through the plates we only wish at this point to remind the reader of the fact that the heat extracted from the lower plate is bigger when the Biot numbers satisfy $B i_{1}<1$, and $B i_{2}>1$, than otherwise.

One can now define the optimal performance of the device as the condition in which the heat extracted from the lower plate is the biggest, while the global entropy production is as small as possible. In order to get it, one only needs to fix the value of $B i_{1}$ between 0.2 and $0.4, B i_{2}$ bigger than unity, and then the Reynolds number should be chosen among the values where the heat transferred through the lower plate has maxima (Figure 7). These are at approximately $R_{\omega}=1.4,2.4,3.4, \ldots$, etc. We know that the global 
Figure 7. Dimensionless heat transported per unit area in lower plate vs. $R_{\omega}$ Solid line: Maxwell fluid, dot-dashed line: Newtonian fluid. $B i_{1}=0.5, B i_{2}=60$ and $\Theta_{A}=5$.

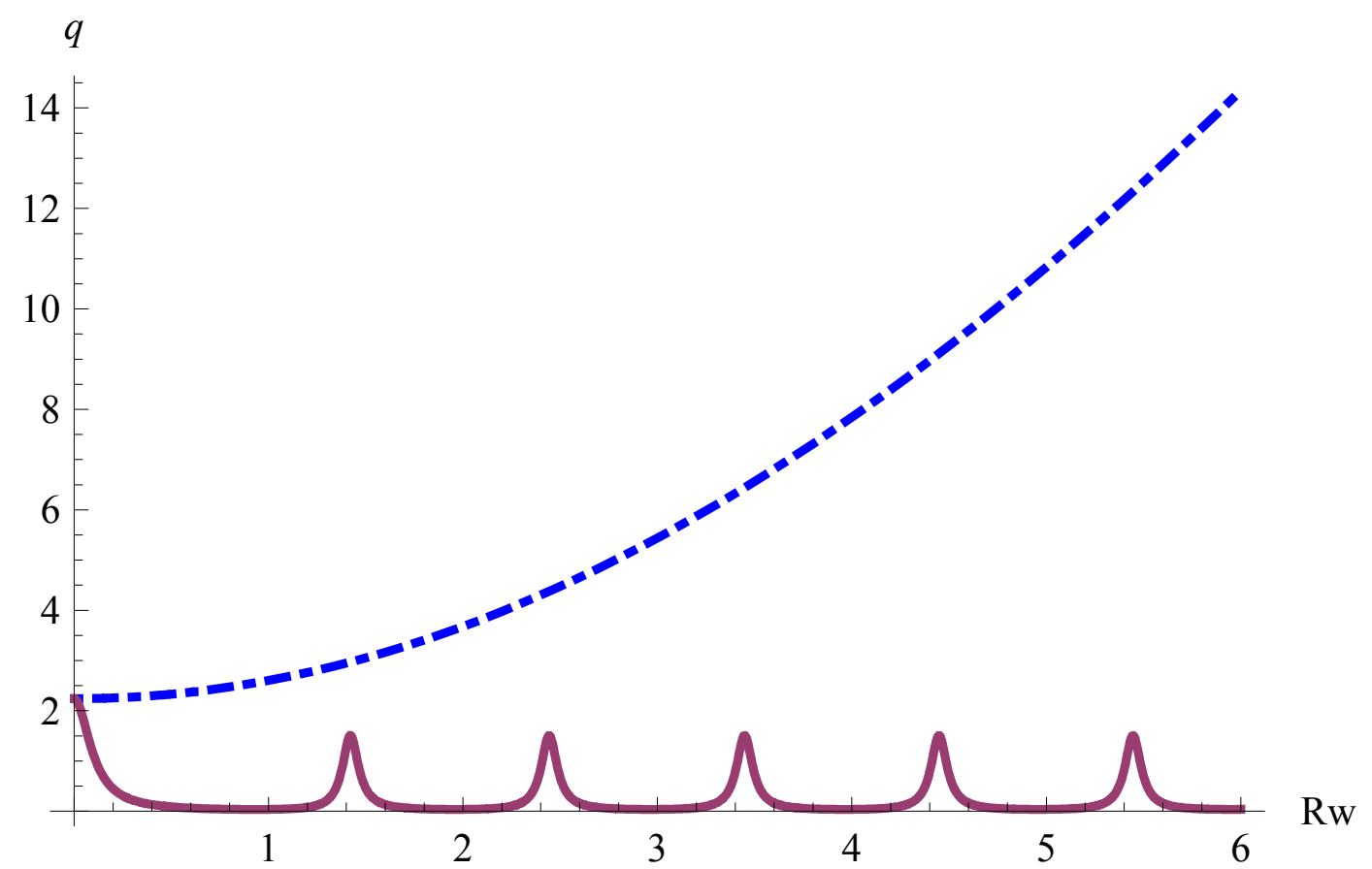

entropy production also has maxima in such points but we now also know that there it is the smallest.

One may rise the question about the use of non-Maxwellian viscoelastic fluids in oscillatory motion for heat transfer applications. Although it may deserve a separate study, in the linear regime other viscoelastic fluids could present a behavior similar to the one found in this paper. Provided viscous and elastic effects are present in a time-periodic flow, a resonant behavior could be expected.

\section{Summary and Conclusion}

In this work we have analyzed the problem of an oscillatory flow of a Maxwell fluid between infinite parallel plates and considered the heat transfer problem with boundary conditions of the third kind. After deriving the velocity and temperature fields, we computed the local and global entropy production in the system. It has been found that for a suitable combination of the physical parameters involved in the oscillatory regime of the problem and those defining the boundary conditions, the system can operate in a minimum global entropy production state. The following is the combination:

1. $0.2<B i_{1}<0.5, B i_{2}>1$

2. $R_{\omega}=1,2,3, \ldots$

The existence of these values of $R_{\omega}$ is an outstanding result that arises from the peculiar properties of the Maxwell fluid (Figures 4 and 7).

The optimal performance of the system has been defined as that in which the heat extracted from the lower plate is a maximum and the global entropy production is as small as possible. We have suggested 
a suitable solution to the problem of reaching such operating state. The first step is to choose the Biot numbers satisfying condition 1 ) above, and then to take $R_{\omega}$ among the values:

3. $R_{\omega}=1.4,2.4,3.4, \ldots$

\section{Acknowledgements}

We acknowledge enlightening discussions with M. López de Haro on the topics of this paper. This work was supported in part by PIFI-IPN México (MAOR). FV thanks financial support from CONACYT and PROMEP, México.

\section{References and Notes}

1. Bejan A., Entropy Generation through Heat and Fluid Flow, Wiley, New York, 1994. 1.

2. Bejan A., Minimization of Entropy Generation, CRC Press, Boca Raton, 1996. 1., 3.1.

3. Chatwin, P.C. On the longitudinal dispersion of passive contaminant in oscillating flows in tubes, $J$. Fluid Mech. 1975, 71 , 513-527. 1.

4. Kurzweg U.H., Enhanced heat conduction in fluids subjected to sinusoidal oscillations, J. Heat Transfer 1985, 107. 459-462. 1.

5. Kurzweg U.H., Enhanced heat conduction in oscillating viscous flows within parallel-plate channels, J. Fluid Mech. 1985, 156, 291-300. 1.

6. López de Haro M., del Río J.A. and Whitaker S., Transport Porous Media 1996, 25, 167. 1.

7. del Río J.A., López de Haro M. and Whitaker S., Enhancement in the dynamic response of a viscoelastic fluid flowing in a tube, Phys. Rev. E 1998, 58, 6323-6327;

8. del Río J.A., López de Haro M. and Whitaker S., Erratum: Enhancement in the dynamic response of a viscoelastic fluid flowing in a tube, Phys. Rev. E 2001, 64 , 039901(E). 1.

9. Tsiklauri D. and Beresnev I., Enhancement in the dynamic response of a viscoelastic fluid flowing through a longitudinally vibrating tube, Phys. Rev. E 2001, 63 , 046304-046308.

10. Castrejón-Pita J.R., del Río J.A., Castrejón-Pita A.A. and Huelsz G., Experimental observation of dramatic differences in the dynamic response of Newtonian and Maxwellian fluids, Phys. Rev. E 2003, 68, 046301-046305. 1.

11. Lambert A.A., Ibáñez G., Cuevas S. and del Río J.A., Optimal behavior of viscoelastic flow at resonant frequencies, Phys. Re. E 70 (2004), 056302; Erratum, Phys. Rev. E 2006, 73, 049902.

12. Yakhot V., Colosqui C., Stokes' second flow problem in a high-frequency limit: application to nanomechanical resonators, J. Fluid Mech. 2007, 586, 249-258. 1., 3.1.

13. Herrera-Velarde, J. R., Zenit, R., Mena, B. Measurements of the temperature rise in non-Newtonian oscillatory pipe flows, J. Non-newtonian Fluid Mech., 2003, 109, 157-176. 1.

14. Kurtcebe, C., Erim, M.Z. Heat transfer of a viscoelastic fluid in a porous channel, Int. J.Heat Mass Transfer 2005, 48, 5072-5077. 1.

15. Pinho, F.T., Celho, P.M. Fully-developed heat transfer in annuli for viscoelastic fluids with viscous dissipation, J. Non-Newtonian Fluid Mech., 2006, 138, 7-21.

16. de Groot S.R. and Mazur P., Non-Equilibrium Thermodynamics, Dover, New York, 1984. 1.

17. Rehage H. and Hoffman R.H., Rheological properties of viscoelastic surfactant systems. J. Phys. 
Chem. 1988, 92, 4712-4719. 1., 3.1.

18. Morrison F. A., Understanding Rheology, Oxford University Press, New York, 2001. 2.4.

19. Ibáñez G., Cuevas S. and López de Haro M., Minimization of entropy generation by asymmetric convective Cooling, Int. J. Heat Mass Transf. 2003, 46 , 1321-1328; 3.1.

20. Ibáñez G., Cuevas S. and López de Haro M., Thermodynamic optimization of radial MHD flow between parallel circular disks, J. Non-Equilib. Thermodyn. 2004, 29, 107-122. 3.1.

(c) 2008 by the authors; licensee Molecular Diversity Preservation International, Basel, Switzerland. This article is an open-access article distributed under the terms and conditions of the Creative Commons Attribution license (http://creativecommons.org/licenses/by/3.0/). 